\title{
REMAINDERS AS INTEGRALS OF PARTIAL DERIVATIVES
}

ARTHUR SARD

1. Introduction. Certain functionals of functions $x=x(s, t)$ of two variables may be written as sums of integrals of partial derivatives of $x$ of order $n$. Indeed there are algorithms for obtaining such integral formulas, and the functionals to which the algorithms apply are readily recognizable. In many applications the functionals are remainders in processes of approximation. For example, among the functionals that we consider later, $F$ is the remainder in the approximation of a double Stieltjes integral; $H$ is the remainder in an interpolation; $G$ and $K$ are the remainders in approximations of the partial derivatives $x_{1,0}(0,0)$ and $x_{1,1}(0,0)$, respectively; $I, J, L$ are remainders in approximate integrations.

The kernel theorem of $\$ 4$ gives a formula for the value $R x$ of a functional $R$ in terms of ordinary (rather than Stieltjes) integrals of partial derivatives of $x$. The table of $\$ 4$ sets forth the full variety of such formulas for the particular functionals $F, \cdots, L$. A few of the formulas are cited explicitly in $\$ \$ 5-8$. Properties of symmetric functionals are stated in $\$ 9$. As regards the use of the kernel theorem of $\$ 4$, the present paper can be read by itself.

The table of $\$ 10$ sets forth the full variety of other formulas for $F, \cdots, L$ given by the mass theorem $(5: 11)$ of an earlier paper ${ }^{1}$ Remainders: functions of several variables. The formulas given by the mass theorem involve Stieltjes integrals of the partial derivatives of $x$.

We shall consider the following nine functionals:

$$
F x=M x\left(s_{0}, t_{0}\right)-\iint_{U} x(s, t) d \gamma(s, t)
$$

where $\gamma(s, t)$ is a function of bounded variation on the rectangle $U: \alpha_{1} \leqq s \leqq \alpha_{2}, \beta_{1} \leqq t \leqq \beta_{2} ; M=\iint_{U} d \gamma(s, t) \neq 0 ; s_{0}=M^{-1} \iint_{U} s d \gamma(s, t)$; $t_{0}=M^{-1} \iint_{U} t d \gamma(s, t)$. As we shall see, $F x$ can be written in terms of second partials of $x$ for a large class of functions $x$. The functionals $F^{*}$ and $F^{* *}$ below are particular cases of $F$ for which integral formulas

Presented to the Society, December 28, 1951; received by the editors March 9 , 1951 and, in revised form, February 11, 1952.

1 Acta Math. vol. 84 (1951) pp. 319-346. We shall refer to this paper as V. All references to ordered pairs of integers, such as $(5: 11)$, are to V. The space $A_{p . q}^{*}$ of the present paper is different from the space $A_{p, q}$ of $\mathrm{V}$. In both papers our definition of function of bounded variation is that of Hardy and W. H. Young (cf. V, p. 321). 
are given explicitly ( $\$ \$ 5$ and 7$)$.

(ii) $\quad F^{*} x=\pi x(0,0)-\iint_{s^{2}+t^{2}<1} x(s, t) d s d t$.

(iii) $F^{* *} x=4 x(0,0)-\int_{-1}^{1} \int_{-1}^{1} x(s, t) d s d t$.

(iv) $\quad G x=[x(1,1)-x(-1,1)+x(1,-1)-x(-1,-1)] / 4$ $-x_{1,0}(0,0)$.

(v) $\quad H x=[x(1,1)+x(-1,1)+2 x(0,-1)] / 4-x(0,0)$.

(vi) $\quad I x=[8 x(0,0)+x(1,1)+x(-1,1)+x(1,-1)+x(-1,-1)] / 3$ $-\int_{-1}^{1} \int_{-1}^{1} x(s, t) d s d t$.

(vii) $\quad J x=\left[12 x(0,0)+x_{1,0}(1,0)-x_{1,0}(-1,0)+x_{0,1}(0,1)\right.$

$$
\left.-x_{0,1}(0,-1)\right] / 3-\int_{-1}^{1} \int_{-1}^{1} x(s, t) d s d t .
$$

(viii) $\quad K x=[x(1,1)-x(-1,1)-x(1,-1)+x(-1,-1)] / 4$

$$
-x_{1,1}(0,0) \text {. }
$$

(ix) $\quad L x=\pi\left[8 x(0,0)+x_{2,0}(0,0)+x_{0,2}(0,0)\right] / 8$

$$
-\iint_{s^{2}+t^{2}<1} x(s, t) d s d t \text {. }
$$

2. The spaces $B_{p, q}$ and $A_{p, e}^{*}$. Keys to integral formulas for the above functionals are certain function spaces $B_{p, q}=B_{p, q}(a, b)$ and $A_{p, \ell}^{*}=A_{p, \ell}^{*}(a, b)$. These spaces are defined relative to a fixed rectangle $U$ of points $(s, t): \alpha_{1} \leqq s \leqq \alpha_{2}, \beta_{1} \leqq t \leqq \beta_{2}$, a fixed point $(a, b)$ in $U$, and fixed nonnegative integers $p, q$. Put $n=p+q$. We first define the core of a function $x=x(s, t)$ in $B_{p, q}$ as the $n+1$ partial derivatives

$$
x_{i, n-i}(a, t), \quad i<p ; \quad x_{p, q}(s, t) ; \quad x_{n-j, j}(s, b), \quad j<q .
$$

(Here and elsewhere indices are nonnegative integers. In particular the class of values $i$ such that $i<p$ is empty if $p=0$.) Each of the partial derivatives (1) is of order $n$.

We now define the space $B_{p, q}$ as the space of all functions $x=x(s, t)$ whose core derivatives (1) exist and are continuous in $t,(s, t), s$, respectively, for $(s, t) \in U$. Thus $B_{p, q}$ is a generalization of the space $C_{n}$ of functions of one variable with continuous $n$th derivative.

In considering a partial derivative we do not always require that 
the order of differentiation be unrestricted. Instead we understand $x_{i, j}$ thus:

$$
x_{i, j}=\frac{\partial^{i+j-\mu-\nu}}{\partial s^{i-\mu} \partial t^{i-\nu}}\left(\frac{\partial^{\mu+\nu} x}{\partial s^{\mu} \partial t^{\nu}}\right), \quad \text { where } \mu=\min (i, p), \nu=\min (j, q) ;
$$

we require here that all orders of the first $\mu+\nu$ differentiations be possible and equivalent and that all orders of the last $i+j-\mu-\nu$ differentiations be possible and equivalent. We describe this understanding about $x_{i, j}$ as the convention of $B_{p, q}$. The convention broadens the meaning of $x_{i, j}$ if $i>p$ or $j>q$. If all orders of differentiation in the core derivatives (1) are possible and equivalent, then a fortiori $x$ is an element of $B_{p, q}$ if the derivatives (1) are continuous.

To illustrate, consider $B_{1,2}$. Its core is

$$
x_{0,3}(a, t), \quad x_{1,2}(s, t), \quad x_{2,1}(s, b), \quad x_{3,0}(s, b) .
$$

Here $x_{2,1}(s, b)$ is understood as $\partial x_{1,1}(s, b) / \partial s$ but not necessarily as $\left[\partial x_{2,0}(s, t) / \partial t\right]_{t=b}$.

For $x$ to be an element of $B_{p, q}$, it is necessary and sufficient that the following derivatives exist and be continuous, $(s, t) \in U$ :

$$
\begin{gathered}
x_{i, j}(s, t), \quad i \leqq p, \quad j \leqq q ; \\
x_{i, j}(a, t), \quad i+j \leqq n, \quad j>q ; \quad x_{i, j}(s, b), \quad i+j \leqq n, \quad i>p .
\end{gathered}
$$

The convention of $B_{p, q}$ applies here.

The space $A_{p, q}^{*}$ is defined, for $p \geqq 1, q \geqq 1$, as the space of those functions $x$ in $B_{p-1, q-1}$ for which the derivatives

$$
x_{i, n-i-1}(a, t), \quad i<p ; \quad x_{n-j-1, j}(s, b), \quad j<q
$$

exist and are continuous in $t$ and $s$ respectively, $(s, t) \in U$. The convention of $B_{p-1, q-1}$ applies here. For $x$ to be an element of $A_{p, \ell}^{*}$, it is necessary and sufficient that the following derivatives exist and be continuous, $(s, t) \in U$ :

$$
\begin{aligned}
& x_{i, j}(s, t), \quad i \leqq p-1, \quad j \leqq q-1 ; \\
& x_{i, j}(a, t), \quad i+j \leqq n-1, \quad j \geqq q ; \\
& x_{i, j}(s, b), \quad i+j \leqq n-1, \quad i \geqq p .
\end{aligned}
$$

Observe that

$$
\begin{gathered}
B_{p, q} \subset A_{p, q}^{*} \subset B_{p-1, q-1}, \quad p q \geqq 1 ; \\
A_{p, q}^{*} \subset A_{p-1, q}^{*}, \quad(p-1) q \geqq 1 ; \quad A_{p, q}^{*} \subset A_{p, q-1}^{*}, \quad p(q-1) \geqq 1 .
\end{gathered}
$$

3. Functionals. A functional $R$ on $A_{p, Q}^{*}$ is a correspondence which 
assigns to each element $x$ of $A_{p, q}^{*}$ a real number $R x$. We shall say that a functional $R$ is admissible $A_{p, q}^{*}$ if $R x$ is a finite sum of LebesgueStieltjes integrals on the derivatives (2); that is, if $R x$ is of the form

$$
\begin{aligned}
R x= & \sum_{i \leqq p-1, j \geqq q-1} \iint_{U} x_{i, j}(s, t) d \gamma^{i, j}(s, t) \\
& +\sum_{i+j \geqq n-1, j \geqq q} \int_{\left[\beta_{1}, \beta_{2}\right]} x_{i, j}(a, t) d \gamma^{i, j}(t) \\
& +\sum_{i+j \geqq n-1, i \geqq p} \int_{\left[\alpha_{1}, \alpha_{2}\right]} x_{i, j}(s, b) d \gamma^{i, j}(s),
\end{aligned}
$$

where the functions $\gamma^{i, j}$ are of bounded variation in their variables.

If $R$ is admissible $A_{p, \varepsilon}^{*}$, then a fortiori $R$ is also admissible $A_{p+1, \varepsilon}^{*}$ and $A_{p, q+1}^{*}$.

Consider the functionals $F, F^{*}, \cdots, L$. (For all of these except $F$, the rectangle $U$ is always assumed large enough to include the points $(-1,-1)$ and $(1,1)$. The point $(a, b)$ is always in $U$, but is otherwise arbitrary unless explicit conditions are placed on $a$ or $b$.) The reader may verify that:

$F, F^{*}, F^{* *}, H, I$ are admissible $A_{1,1}^{*}(a, b)$;

$G$ is admissible $A_{1,1}^{*}(a, 0)$, also $A_{2,1}^{*}(a, b)$;

$J$ is admissible $A_{1,1}^{*}(0,0)$, also $A_{1,2}^{*}(a, 0)$, also $A_{2,1}^{*}(0, b)$, also $A_{2,2}^{*}(a, b)$;

$K$ is admissible $A_{1,2}^{*}(a, 0)$, also $A_{2,1}^{*}(0, b)$, also $A_{2,2}^{*}(a, b)$;

$L$ is admissible $A_{1,2}^{*}(0,0)$, also $A_{2,1}^{*}(0,0)$, also $A_{1,3}^{*}(a, 0)$, also $A_{3,1}^{*}$ $(0, b)$, also $A_{3,3}^{*}(a, b)$.

These functionals are not admissible $A_{p, \ell}^{*}(a, b)$ except for the instances cited above and instances derivable therefrom by increasing subscripts $p$ or $q$.

We say that a functional $R$ is zero for degree $m$ if $R x=0$ whenever $x=x(s, t)$ is a polynomial in $(s, t)$ of degree $m$. Suppose that $R$ is admissible $A_{p, q}^{*}$ for some $p, q$. Then $R$ will be zero for degree $m$ if and only if $R x=0$ when $x$ is each of the $(m+1)(m+2) / 2$ polynomials $1, s, t, s^{2}, s t, t^{2}, \cdots, s^{m}, s^{m-1} t, s^{m-2} t^{2}, \cdots, t^{m}$. If $R$ is zero for degree $m$, then $R$ is also zero for degree $m-1$.

Thus:

$F, F^{*}, F^{* *}, H$ are zero for degree 1 ;

$G$ is zero for degree 2 ;

$I, J, K, L$ are zero for degree 3 .

Each of these functionals is not zero for degree greater than that cited, except possibly $F$ for particular functions $\gamma$. 
4. Kernel theorem. Suppose that the functional $R$ is admissible $A_{p, q}^{*}$ and zero for degree $n-1$, where $n=p+q, p q \geqq 1$. Then there exist functions $f^{(i)}(t), i<p ; g(s, t) ; h^{(j)}(s), j<q$; all of bounded variation, such that, for all $x$ in $B_{p, q}$,

$$
\begin{aligned}
R x= & \sum_{i<p} \int_{\beta_{1}}^{\beta_{2}} x_{i, n-i}(a, t) f^{(i)}(t) d t+\int_{\beta_{1}}^{\beta_{2}} \int_{\alpha_{1}}^{\alpha_{2}} x_{p, q}(s, t) g(s, t) d s d t \\
& +\sum_{j<q} \int_{\alpha_{1}}^{\alpha_{2}} x_{n-j, j}(s, b) h^{(j)}(s) d s .
\end{aligned}
$$

Conversely, given kernels $f^{(i)}(t), g(s, t), h^{(j)}(s)$ of bounded variation, the functional $R$ defined by (4) can be extended onto $A_{p, q}^{*}$ so as to be admissible $A_{p, Q}^{*}$ and zero for degree $n-1$. The kernels may be determined from the following relations:

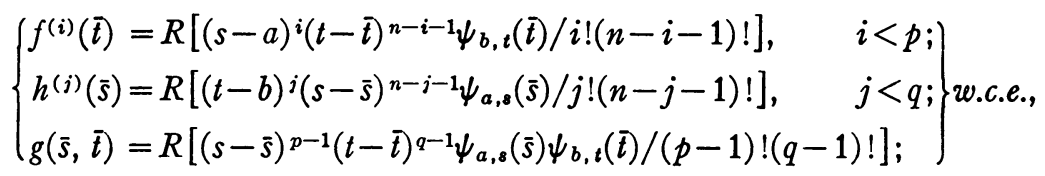

where

$$
\psi_{a, s}(\bar{s})=\left\{\begin{aligned}
1 & \text { if } a \leqq \bar{s}<s \\
-1 & \text { if } s \leqq \bar{s}<a \\
0 & \text { otherwise. }
\end{aligned}\right.
$$

In (5) the functional $R$ operates on its arguments as functions of $s, t$; the quantities $\bar{s}, \bar{t}$ are parameters. The letters w.c.e. are an abbreviation for "with countable exceptions." What is meant is that the first formulas of (5) hold except possibly for countably many values of $\bar{s}$ and $\bar{t}$ and that the last formula holds except possibly on countably many line segments parallel to the $\bar{s}$ axis and on countably many line segments parallel to the $\vec{t}$ axis. Knowledge of the kernels w.c.e. is sufficient for their use in (4).

The above theorem will be proved in $\$ 11$.

The formula (4) expresses $R x$ in terms of the core of $x$ in $B_{p, q}$ and gives us a powerful insight into the nature of $R$. For $R$ to be admissible $A_{p, q}^{*}$, it is not necessary that $R$ involve all of $U$. One might say, then, that $R$ need not have a rectangular character. Whenever $x \in B_{p, q}$, however, $R x$ can be written in the form (4), providing that $R$ satisfies the hypothesis of the theorem. Thus a large part of the weight of what must be known falls on $x$ rather than on $R$.

Efficient appraisals may be based on (4) (see $\$ 8$ of V). The terms of (4) cannot be combined more simply, as there are functions $x$ in 
$B_{p, q}$ for which any one and only that one of the $n+1$ core derivatives is not zero.

In the following table we list all values of $a, b, p, q$ for which the functionals $F, \cdots, L$ satisfy the hypothesis on $R$ of the above kernel theorem.

TABLE

Complete list of cases in which the kernel theorem applies to $F, \cdots, L$.

\begin{tabular}{|c|c|c|c|c|c|c|c|}
\hline$R^{\prime} F, F^{*}, F^{* *}$, or $H$ & $G$ & $I$ & & $J$ & & $K$ & $L$ \\
\hline$p$ & 121 & $\begin{array}{lllll}1 & 1 & 2 & 1 & 23\end{array}$ & 112 & 213 & 12 & 213 & 1213 \\
\hline 1 & 112 & $\begin{array}{lllll}12 & 1 & 3 & 2 & 1\end{array}$ & 121 & 231 & 21 & 31 & 21331 \\
\hline$a$ & & & $\begin{array}{ll}0 & 0\end{array}$ & 0 & 0 & 0 & $\begin{array}{llll}0 & 0 & 0\end{array}$ \\
\hline$b$ & 0 & & 00 & 0 & 0 & 0 & 000 \\
\hline
\end{tabular}

We give certain explicit instances of (4) in the following sections. Formulas for $G$ are given in $\$ 7$ of $\mathrm{V}$. The reader may work out any of the formulas indicated in the above table.

It is interesting to compare the present kernel theorem with theorem $(6: 11)$ of $\mathrm{V}$. In the new theorem $R x$ may involve $x_{p-1, q}(a, t)$ and $x_{p, q-1}(s, b)$, derivatives which are not in the core of the space $A_{p, q}$ of $V$. In many applications this is a material advantage. ${ }^{2}$ The new theorem may be used, however, only when $R$ is known to be admissible $A_{p, q}^{*}$. In a later paper we shall prove the following. Let the norm of $x$ in $A_{p, q}^{*}$ be the maximum of the maxima of the absolute values of the derivatives $(2),(s, t) \in U$. A functional $R$ is linear on $A_{p, \varepsilon}^{*}$ if and only if $R$ is equal, on $A_{p, \varepsilon}^{*}$, to a functional admissible $A_{p, \varepsilon}^{*}$.

5. A formula for $F^{* *}$. For $x \in B_{1,1}(0,0)$,

$$
\begin{aligned}
F^{* *} x= & \int_{-1}^{1} x_{0,2}(0, t) f(t) d t+\int_{-1}^{1} \int_{-1}^{1} x_{1,1}(s, t) g(s, t) d s d t \\
& +\int_{-1}^{1} x_{2,0}(s, 0) h(s) d s
\end{aligned}
$$

where

$$
\begin{gathered}
f(t)=-(1-|t|)^{2}, \quad h(s)=f(s), \\
g(s, t)=-g(-s, t)=-g(s,-t)=g(-s,-t)=-(1-s)(1-t), \\
0<s, t \leqq 1 .
\end{gathered}
$$

The values of $g(s, t)$ when $s=0$ or $t=0$ are immaterial. Formulas of

2 A functional which is admissible $A_{p, q}^{*}$ without being zero for degree $n-1$ can be converted into one which satisfies the hypothesis of the present kernel theorem (cf. (6:18)). 
the above type can be verified by direct integration.

6. A formula for $H$. For $x \in B_{1,1}(0,1)$,

$$
H x=\int_{-1}^{1} x_{0,2}(0, t) f(t) d t+\int_{-1}^{1} x_{2,0}(s, 1) h(s) d s,
$$

where $2 f(t)=1-|t|, 2 h(s)=f(s)$.

7. A formula for $F^{*}$. For $x \in B_{1,1}(0,0)$,

$$
\begin{aligned}
F^{*} x= & \int_{-1}^{1} x_{0,2}(0, t) f(t) d t+\int_{-1}^{1} \int_{-1}^{1} x_{1,1}(s, t) g(s, t) d s d t \\
& +\int_{-1}^{1} x_{2,0}(s, 0) h(s) d s
\end{aligned}
$$

where

$$
\begin{aligned}
f(t) & =f(-t)=-2 \int_{t}^{1}(\tau-t)\left(1-\tau^{2}\right)^{1 / 2} d \tau, 0 \leqq t \leqq 1 ; \\
h(s) & =f(s) ; \\
g(s, t) & =-g(-s, t)=-g(s,-t)=g(-s,-t) \\
& =-\int_{0}^{(1-t)^{1 / 2}} d \sigma \int_{t}^{\left(1-\sigma^{2}\right)^{1 / 2}} d \tau \\
& =g(t, s) \text { if } 0<s, t \text { and } s^{2}+t^{2}<1, \\
g(s, t) & =0 \text { if } s^{2}+t^{2} \geqq 1 .
\end{aligned}
$$

8. A formula for $I$. For $x \in B_{1,3}(0,0)$,

$$
\begin{aligned}
I x= & \int_{-1}^{1} x_{0,4}(0, t) f(t) d t+\int_{-1}^{1} \int_{-1}^{1} x_{1,3}(s, t) g(s, t) d s d t \\
& +\int_{-1}^{1} x_{2,2}(s, 0) h^{(2)}(s) d s+\int_{-1}^{1} x_{3,1}(s, 0) h^{(1)}(s) d s \\
& +\int_{-1}^{1} x_{4,0}(s, 0) h^{(0)}(s) d s
\end{aligned}
$$

where

$$
\begin{aligned}
36 f(t) & =(1-|t|)^{3}(1+3|t|) ; \quad 6 h^{(2)}(s)=1-s^{2} ; & & h^{(1)}(s)=0 ; \\
g(s, t) & =-g(-s, t)=-g(s,-t)=g(-s,-t) & & h^{(0)}(s)=f(s) ; \\
& =(1-t)^{2}(s+t-s t) / 6, & & 0<s, t \leqq 1 .
\end{aligned}
$$


9. Symmetry. Suppose that the fundamental rectangle $U$, the point $(a, b)$, and the functional $R$ of the kernel theorem are symmetric relative to the $t$ axis:

$$
\alpha_{2}=-\alpha_{1} ; \quad a=0 ; \quad R[x(s, t)]=R[x(-s, t)], \quad x \in A_{p, q}^{*}(0, b) .
$$

Then each kernel in (4) that involves $s$ is even or odd in $s$ according as the number of differentiations with respect to $s$ in the corresponding partial derivative of $x$ is even or odd.

Likewise for skew symmetry: If

$$
\alpha_{2}=-\alpha_{1} ; \quad a=0 ; \quad R[x(s, t)]=-R[x(-s, t)], \quad x \in A_{p, q}^{*}(0, b) ;
$$

then each kernel that involves $s$ is even or odd in $s$ according as the number of differentiations is odd or even.

The dual assertions (with $s$ and $t$ interchanged) also are true.

10. Other integral formulas. In addition to the formulas of type (4), there exist formulas of type (5:12) which express $R x$ as a sum of Stieltjes integrals on the derivatives of $x$ in the core of $B_{p, q}$. Such mass formulas are often useful in the theory of approximation. Each formula (4) is of the type $(5: 12)$, but a formula $(5: 12)$ is equivalent to a formula (4) with integrable kernels if and only if the measures corresponding to the masses $(5: 13)$ are absolutely continuous.

In the following table we list all values of $a, b, p, q$, other than the values in the table of $\$ 4$, for which the functionals $F, \cdots, L$ satisfy the hypothesis on $R$ of the mass theorem $(5: 11)$.

TABLE

Complete list of cases, other than those of the table of $\$ 4$, in which mass formulas $(5: 12)$ exist for $F, \cdots, L$

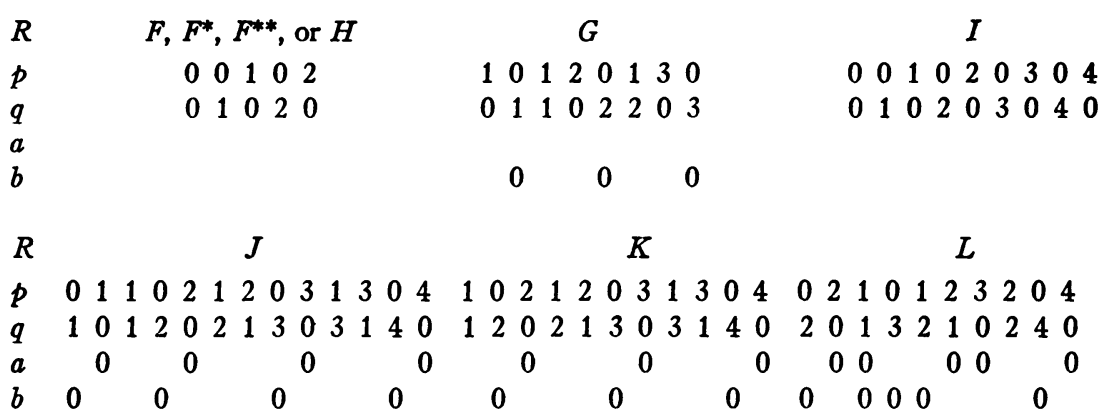

11. Proof of the theorem of $\$ 4$. Define $s^{k}$ for integral $k$ as follows: $s^{k}=0$ if $k<0, s^{0}=1, s^{k}=s^{k-1} / k$ if $k>0$. Consider $x \in B_{p, q}$; we may write 
Taylor's formula (5:4) for $x$. Since $R$ is zero for degree $n-1$ and additive, $R x=R y$, where

$$
\begin{aligned}
y= & \sum_{i<p}(s-a)^{i} \int_{b}^{t}(t-\bar{t})^{n-i-1} x_{i, n-i}(a, \bar{t}) d \bar{t} \\
& +\int_{b}^{t} \int_{a}^{s}(s-\bar{s})^{p-1}(t-\bar{t})^{q-1} x_{p, q}(\bar{s}, \bar{t}) d \bar{s} d \bar{t} \\
& +\sum_{j<q}(t-b)^{j} \int_{a}^{s}(s-\bar{s})^{n-j-1} x_{n-j, j}(\bar{s}, b) d \bar{s} .
\end{aligned}
$$

To evaluate $R y$ we use (3) and Fubini's theorem. Because of the convention of $B_{p-1, q-1}$ as to differentiation,

$$
\begin{aligned}
y_{\kappa, \lambda}(s, t)= & \sum_{i<p}(s-a)^{i-\kappa} \int_{b}^{t}(t-\bar{t})^{n-i-1-\lambda} x_{i, n-i}(a, \bar{t}) d \bar{t} \\
& +\int_{b}^{t} \int_{a}^{s}(s-\bar{s})^{p-1-\kappa}(t-\bar{t})^{q-1-\lambda} x_{p, q}(\bar{s}, \bar{t}) d \bar{s} d \bar{t} \\
& +\sum_{i<q}(t-b)^{i-\lambda} \int_{a}^{s}(s-\bar{s})^{n-i-1-\kappa} x_{n-j, j}(\bar{s}, b) d \bar{s} \\
= & \sum_{i<p}(s-a)^{i-\kappa} \int_{(t-\bar{t})^{n-i-1-\lambda} \psi_{b, t}(\bar{t}) x_{i, n-i}(a, \bar{t}) d \bar{t}} \\
& +\iint(s-\bar{s})^{p-1-\kappa}(t-\bar{t})^{q-1-\lambda} \psi_{a, 8}(\bar{s}) \psi_{b, t}(\bar{t}) x_{p, q}(\bar{s}, \bar{t}) d \bar{s} d \bar{t} \\
& +\sum_{j<q}(t-b)^{j-\lambda} \int(s-\bar{s})^{n-j-1-\kappa} \psi_{a, s}(\bar{s}) x_{n-j, j}(\bar{s}, b) d \bar{s}, \\
y_{\kappa, \lambda}(a, t)= & \frac{\partial^{\lambda-q+1}}{\partial t^{\lambda-q+1}} y_{\kappa, q-1}(a, t)=\int_{b}^{t}(t-\bar{t})^{n-\kappa-1-\lambda} x_{\kappa, n-\kappa}(a, \bar{t}) d \bar{t}+0+0 \\
= & \int(t-\bar{t})^{n-\kappa-1-\lambda} \psi_{b, t}(\bar{t}) x_{\kappa, n-\kappa}(a, \bar{t}) d \bar{t}, \kappa+\lambda \leqq n-1, \lambda \geqq q ; \\
y_{\kappa, \lambda}(s, b)= & \int(s-\bar{s})^{n-\lambda-1-\kappa} \psi_{a, s}(\bar{s}) x_{n-\lambda, \lambda}(\bar{s}, \bar{b}) d \bar{s}, \kappa+\lambda \leqq n-1, \kappa \geqq p ;
\end{aligned}
$$

where the limits of integration, when not given, are $\alpha_{1}, \alpha_{2}$ for $\bar{s}$ and $\beta_{1}, \beta_{2}$ for $\bar{t}$. It follows that (4) holds with 
(6)

$$
\begin{array}{rlr}
f^{(i)}(\bar{t})= & \sum_{\kappa \leqq p-1, \lambda \leqq q-1} \iint(s-a)^{i-\kappa}(t-\bar{t})^{n-i-1-\lambda} \psi_{b, t}(\bar{t}) d \gamma^{\kappa, \lambda}(s, t) \\
& +\sum_{q \leqq \lambda \leqq n-i-1} \int(t-\bar{t})^{n-i-1-\lambda} \psi_{b, t}(\bar{t}) d \gamma^{i, \lambda}(t), & i<p ; \\
h^{(j)}(\bar{s})= & \text { the dual expression, } \\
g(\bar{s}, \bar{t})= & \sum_{\kappa \leqq p-1, \lambda \leqq q-1} \iint(s-\bar{s})^{p-1-\kappa}(t-\bar{t})^{q-1-\lambda} \psi_{a, s}(\bar{s}) \psi_{b, t}(\bar{t}) \\
& \cdot d \gamma^{\kappa, \lambda}(s, t) ;
\end{array}
$$

these relations being valid at least almost everywhere $\vec{t}, \bar{s},(\bar{s}, \bar{t})$, respectively.

Now the second members of (5) are defined w.c.e. and equal, whenever defined, the respective functions (6). For, $\psi_{a, s}(\bar{s})$, as a function of $s$, is constant except for a jump of unity at $s=\bar{s}$. Hence the $s$ derivative of $(s-\bar{s})^{k} \psi_{a, s}(\bar{s})$ is $(s-\bar{s})^{k-1} \psi_{a, s}(\bar{s})$ except possibly at $s=\bar{s}$. Hence all the derivatives that enter in the evaluation of the second members of (5) by (3) exist and are continuous, except possibly at $s=\bar{s}$ or $t=\bar{t}$. Hence the second members exist except possibly when $\bar{s}$ or $\bar{t}$ is a discontinuity of one of the functions $\gamma^{i, j}$; that is, the second members exist w.c.e. The reader may transform (5) into (6), by means of (3) and the relation $\psi_{a, a}(\bar{s})=0$. Study of (6) shows that the kernels $f^{(i)}, g, h^{(j)}$, when properly defined at the exceptional arguments $\bar{s}, \bar{t}$, are functions of bounded variation.

To establish the converse part of the theorem, we integrate each term of (4) by parts (cf. the last equation of page 338 of V). Such integration is surely possible, since the kernels are of bounded variation.

Queens College 